TAIWANESE JOURNAL OF MATHEMATICS

Vol. 7, No. 2, pp. 275-281, June 2003

This paper is available online at http://www.math.nthu.edu.tw/tjm/

\title{
A CUBIC SYSTEM WITH AN INVARIANT TRIANGLE SURROUNDING AT LEAST ONE LIMIT CYCLE
}

\author{
Zhenhai Liu, E. Sáez, and I. Szántó
}

\begin{abstract}
We show that the existence of a cubic polynomial systems having three real invariant straight lines forming a triangle with at least one limit cycle in the interior.
\end{abstract}

\section{INTRODUCTION}

Consider the following polynomial system on the plane.

$$
\begin{aligned}
& \dot{x}=P(x, y)=\sum_{i+j=0}^{n} a_{i j} x^{i} y^{j}, \\
& \dot{y}=Q(x, y)=\sum_{i+j=0}^{n} b_{i j} x^{i} y^{j}, \text { with } a_{i j}, b_{i j} \in \mathbb{R} .
\end{aligned}
$$

The problem of analyzing periodic solutions has been widely studied and, consequently, there is extensive literatures on this subject. This activity reflects the breadth of interests in Hilbert's 16th problem and the fact that such systems are often used in mathematical models. Suppose that the origin of (1) is a critical point of center-focus type. We are concerned with two closely related questions, both of which are significant elements in work on Hilbert's 16th problem. The first is the number of limit cycles (that is, isolated periodic solutions) which bifurcate from a critical point and the second is the derivation of necessary and sufficient conditions

Received January 23, 2002; revised April 9, 2002.

Communicated by S. N. Chow.

2000 Mathematics Subject Classification: 92D25, 34C, 58F14, 58F21.

Key words and phrases: Limit cycles, Polynomial systems, Existence results.

Financed partially by: NNSF of China Grant No.10171008, Education Department of China Grant No.01084, Hunan Education Department of China Grant No.01A025, USM Grant No.120121 and Fondecyt Grant 1000015. 
for a critical point to be a center (that is, all orbits in the neighborhood of the critical point are closed).

In order to describe Hilbert's 16th problem more precisely, let $S_{n}$ be the collection of systems of form (1), with $P$ and $Q$ of degree at most $n$, and let $\pi(P, Q)$ be the number of limit cycles of (1). We let $(P, Q)$ denote system (1). Define the so-called Hilbert numbers by

$$
H_{n}=\operatorname{Sup}\left\{\pi(P, Q) ;(P, Q) \in S_{n}\right\} .
$$

The problem consists of estimating $H_{n}$ in terms of $n$ and obtaining the possible relative configurations of limit cycles. This is the second part of the 16th problem, which is contained in the famous list of problems proposed by Hilbert at the International Congress of Mathematicians held in Paris in 1900.

\section{Limit Cycles And Centre Conditions}

To explain the way in which the twin questions of bifurcation and integrability are approached, we suppose that the origin is a critical point of (1) and transform the system to canonical form:

$$
\dot{x}=\lambda x+y+p(x, y), \quad \dot{y}=-x+\lambda y+q(x, y),
$$

where $p, q$ are polynomials without linear terms. For the origin to be a centre we must have $\lambda=0$. If $\lambda=0$ and the origin is not a centre, it is said to be a fine focus.

The necessary conditions for a centre are obtained by computing the focal values. These are polynomials in the coefficients arising in $P$ and $Q$ and are defined as follows. There is a function $V$, analytic in a neighbourhood of the origin, such that the rate of change along orbits, $\dot{V}$, is of the form $\eta_{2} r^{2}+\eta_{4} r^{4}+\cdots$, where $r^{2}=x^{2}+y^{2}$. The focal values are the $\eta_{2 k}$, and the origin is a centre if and only if they are all zero. However, since they are polynomials, the ideal they generate has a finite basis, so there is $M$ such that $\eta_{2 \ell}=0$, for $\ell \cdot M$, implies that $\eta_{2 \ell}=0$ for all $\ell$. The value of $M$ is not known a priori, so it is not clear in advance how many focal values should be calculated.

The software Mathematica [11] is used to calculate the first few focal values. These are then 'reduced' in the sense that each is computed modulo the ideal generated by the previous ones: that is, the relations $\eta_{2}=\eta_{4}=\cdots=\eta_{2 k}=0$ are used to eliminate some of the variables in $\eta_{2 k+2}$. The reduced focal value $\eta_{2 k+2}$, with strictly positive factors removed, is known as the Liapunov quantity $L(k)$. Common factors of the reduced focal values are removed and the computation proceeds until it can be shown that the remaining expressions cannot be zero simultaneously. 
The circumstances under which the calculated focal values are zero yield the necessary centre conditions. The origin is a fine focus of order $k$ if $L(i)=0$ for $i=0,1, \ldots, k-1$ and $L(k) \neq 0$. At most $k$ limit cycles can bifurcate out of a fine focus of order $k$; these are called small amplitude limit cycles.

Various methods used to prove the sufficiency of the possible centre conditions. Of particular interest to us in this paper is the construction of an integrating factor - that is, a function $B$ such that

$$
\frac{\partial}{\partial x}(B P)+\frac{\partial}{\partial y}(B Q)=0
$$

$\ldots$ of the form $\ell_{1}^{\alpha_{1}} \ell_{2}^{\alpha_{2}} \ldots \ell_{k}^{\alpha_{k}}$ where each $\ell_{k}$ is of the form $a_{k} x+b_{k} y+1$. If such a function exists, the origin is a centre.

We consider systems in which $P$ and $Q$ both factorizable and having at least one real invariant straight lines, where the real straight line $a x+b y+c=0$ is invariant for the flow of (1) and is called an a real invariant straight line of (1) if

$$
a P(x, y)+b Q(x, y)=(a x+b y+c) R(x, y)
$$

for some real polynomial $R$.

It is well known that the existence of invariant straight lines of (1) restricts the number of limit cycles.

Let $S_{n, l}$ be the collection of systems (1) having $l$ real invariant straight lines. As we denote $\pi(P, Q)$ as the number of limit cycle of (1) we can define

$$
H_{n}(l)=\operatorname{Sup}\left\{\pi(P, Q) ;(P, Q) \in S_{n, l}\right\}
$$

At present, the following results are known

$$
\begin{array}{rlll}
H_{2}(2) & = & \text { N. N. Bautin [1] } \\
H_{2}(1) & \cdot & 1 & \text { L. A. Cherkas and L. I. Zhilevich [2] } \\
H_{3}(5) & =0 & \text { D. Guoren and W.Songlin [3] } \\
H_{n}\left(\frac{(n-1)(n+2)}{2}\right)= & 0 & \text { S. Guangjian and S.Jifang [8] } \\
H_{3}(4) & \cdot & 1 & \text { R. Kooij [4] } \\
H_{3}(2) \geq & 4 & \text { N. G. Lloyd, J. M. Pearson, E. Sáez, and I. Szántó [5] } \\
H_{3}(2) \geq & 6 & \text { N. G. Lloyd, J. M. Pearson, E. Sáez, and I. Szántó [6] } \\
H_{3}(3) \geq & 2 & \text { Y. Yanqian and Y. Weiyin [10] } \\
H_{3}(3) \geq & 4 & \text { E. Sáez, I. Szántó and E. Gonzalez-Olivares [7] }
\end{array}
$$

Ye Yanqian in the paper [9] considers a kind of cubic systems with an invariant triangle whose sides are on real invariant straight lines and whose vertices are 
saddles. Under certain conditions on the coefficients, the relative positions of other critical points of the cubic system and its invariant straight lines with respect to the invariant triangle are determined. In all of the cases, the singular point at the interior of the triangle is an hyperbolic focus.

In this paper we will consider a class of cubic vector field given by the following system:

(2) $X_{\mu}:\left\{\begin{array}{c}\dot{x}=(-1+a b x)\left(-c x-\left(a^{2}+a b-a b c-b^{2} c\right) x^{2}+y+\left(2 a^{2}-\right.\right. \\ \left.\left.a b+a^{2} b-b^{2}+a b^{2}-a b c\right) x y+\left(a b-2 a^{2} b\right) y^{2}\right) \\ \dot{y}=(-1+a b y)\left(-x+\left(a^{2}+a b^{2}+b^{3}\right) x y-\left(a^{2} b+a b^{2}\right) y^{2}\right)\end{array}\right.$

where $\mu=(a, b, c) \in \mathbb{R}^{3}$

We shall show that there are systems of this form having three real invariant straight lines forming a triangle surrounding at least one limit cycle, where the small amplitude limit cycle are limit cycles which bifurcate out of a nonhyperbolic focus.

\section{MAIN Results}

We use the Mathematica software to calculate the focal values for (2) and we obtain the Liapunov quantity $L(i)$ from the corresponding focal value $\eta_{2 i+2}$ as described above. The objective is to find a basis of polynomials $L(i)$ from the focal values and obtaining a set of necessary centre conditions for the system. The sufficiency of these conditions is confirmed by constructing integrating factors that are products of powers of real invariant straight lines.

It is clear that for $a b \neq 0$, the system (2) has three real invariant straight lines, namely,

$$
x=\frac{1}{a b}, \quad y=\frac{1}{a b} \text { and } \frac{(a+b)}{a} x-y-\frac{1}{a b}=0
$$

If $b<0, a>0$ and $a+b<0$, the above straight lines forming a triangle surrounding the origin.

The linear part of $(2)$ at the singularity $(0,0)$ is

$$
D X_{\mu}(0,0)=\left(\begin{array}{cc}
c & -1 \\
1 & 0
\end{array}\right)
$$

If $c=0$, we consider $\tilde{\mu}=(a, b, 0)$, and we have that $\operatorname{div} X_{\tilde{\mu}}(0,0)=0$ and $\operatorname{det} D X_{\tilde{\mu}}(0,0)=1$, then the critical point $(0,0)$ is a fine focus.

Let us consider $\Sigma=\{(a, b, c) \mid a>0, b<0, a+b<0\}, \pi: \Sigma \rightarrow \mathbb{R}^{2}$ the projection $\pi(a, b, c)=(a, b)$ and $f(a, b)=b^{3}-a\left(1+2 b-b^{2}\right)$ where $(a, b) \in \mathbb{R}^{2}$. 
Lemma. Let $(a, b) \in \pi(\Sigma) \backslash f^{-1}(0)$. The vector field $X_{\tilde{\mu}}$ has at the singularity $(0,0)$ a fine focus of order one:

$$
\left\{\begin{array}{rl}
\text { repelling } & \text { if } \quad(a, b) \in f^{-1}(0, \infty) \\
\text { attracting } & \text { if } \quad(a, b) \in f^{-1}(-\infty, 0)
\end{array} .\right.
$$

Proof. Let $(a, b) \in \pi(\Sigma) \backslash f^{-1}(0)$. If $c=0$ then $L(0)=0$. Using the software Mathematica [11] we obtain the second Liapunov quantity

$$
L(1)=a\left(-a-2 a b+a b^{2}+b^{3}\right)=a f(a, b) .
$$

Then $\operatorname{sgn}(L(1))=\operatorname{sgn}(f(a, b))$ and the proof follows.

Theorem 1. The critical point $(0,0)$ of $(2)$ is a center if and only if $c=0$ and $(a, b) \in f^{-1}(0) \cap \pi(\Sigma)$.

Proof. Let us assume that the origin of (2) is a centre. Then $L(0)=0$ and follows that $c=0$. As $L(1)=a f(a, b), a>0$ and $L(1)=0$ we have that $f(a, b)=0$.

To prove that the conditions are sufficient we construct the integrating factor of $X_{\tilde{\mu}}$ from invariant lines $x=\frac{1}{a b}, y=\frac{1}{a b}$ and $y=\frac{(a+b)}{a} x-\frac{1}{a b}$.

If $(a, b) \in f^{-1}(0) \cap \pi(\Sigma)$ and $c=0$, it is easy to prove that $\phi_{b}(x, y)=$ $\left(x-\frac{1}{a b}\right)^{a_{1}}\left(y-\frac{1}{a b}\right)^{a_{2}}\left(\frac{(a+b)}{a} x-y-\frac{1}{a b}\right)^{a_{3}}$ is an integrating factor, where

$$
\begin{aligned}
& a_{1}=\left(-2-8 b-5 b^{2}+7 b^{3}-b^{4}\right) /\left(-1-2 b+b^{2}\right)^{2} \\
& a_{2}=\left((1+2 b)\left(-1-4 b-2 b^{2}+6 b^{3}-2 b^{4}\right)\right) /\left(b\left(-1-2 b+b^{2}\right)^{2}\right) \\
& a_{3}=\left((1+2 b)\left(-1-2 b+3 b^{2}-b^{3}\right)\right) /\left(-1-2 b+b^{2}\right)^{2}
\end{aligned}
$$

and the proof of the Theorem 1 now follows.

Theorem 2. In the parameter space $\Sigma$, there are two slice regions

$$
\begin{aligned}
& S_{1}=\left\{(a, b, c):(a, b) \in \pi(\Sigma) \cap f^{-1}(0, \infty),-\epsilon<c<0\right\} \\
& S_{2}=\left\{(a, b, c):(a, b) \in \pi(\Sigma) \cap f^{-1}(-\infty, 0), 0<c<\epsilon\right\}
\end{aligned}
$$

for some small $\epsilon$, such that for all $\mu \in S_{1} \cup S_{2}$, the differential system (2) has exactly three invariant straight lines forming a triangle, and at least one hyperbolic small amplitude limit cycle surrounding the singularity $(0,0)$ in the interior of the triangle. 
Proof. By (3) the vector field $X_{\mu}$ has a triangle surrounding the origin, whose sides are on the invariant straight lines.

Let us consider $0<\epsilon<<1,(a, b) \in \pi(\Sigma) \backslash f^{-1}(0)$ and $(a, b) \in \pi(\Sigma) \cap$ $f^{-1}(0, \infty)$ (respectively $\left.(a, b) \in \pi(\Sigma) \cap f^{-1}(-\infty, 0)\right)$. By the Lemma the vector field $X_{\mu}$ with $\mu=(a, b, 0)$ has a repelling fine focus of order one (respectively an attracting fine focus of order one ). If $-\epsilon<c<0$ (respectively $0<c<\epsilon$ ) the stability at the origin of $X_{\mu}$ is reversed and a hyperbolic attracting (respectively repelling) small amplitude limit cycle is created (Hopf Bifurcation)

\section{ACKNOWLEDGMENTS}

The authors would like to thank the referee for useful comments and suggestions.

\section{REFERENCES}

1. N. N. Bautin, On Periodic solutions of a systems of differential equations., Prikl. Math. i. Mech 18 (1954), 128. (Russian)

2. L. A. Cherkas and L. I. Zhilevich, The limit cycles of certain differential equations, Differentsial'nye Uravneniya 8 (1972), 1207-1213. (Russian)

3. Dai Guoren and Wo Songlin, Closed orbits and straight line invariants in $E_{3}$ systems, Acta Mathematica Scientia, 9 (1989), 3, 251-261. (Chinese)

4. R. Kooij, Limit cycles in polynomial systems, Thesis, University of Technology, Delft (1993).

5. N. G. Lloyd, J. M Pearson, E. Sáez, and I. Szántó, Limit cycles of a cubic Kolmogorov system, Applied Mathematics Letters, 9 (1996), pp.15-18.

6. N. G. Lloyd, J. M Pearson, E. Sáez, and I. Szántó, I. A cubic Kolmogorov system with six limit cycles, To appear in Computers and mathematics with applications.

7. E. Sáez, I. Szántó \& E. González-Olivares, Cubic Kolmogorov system with four limit cycles and three invariant straight lines, Nonlinear Analysis, 47(7) (2001), 4521-4525.

8. Suo Guangjian and Sun Jifang, The n-degree differential system with $\frac{(n-1)(n+2)}{2}$ straight line solutions has no limit cycles, Proc. of ordinary Differential Equations and Control Theory, Wuhan (1987), 216-220. (Chinese)

9. Ye Yanqian, Cubic Kolmogorov system having three straight line integrals forming a triangle, Ann. of Diff. Eqs. 6(3) (1990), 365-372.

10. Ye Yanqian and Ye Weiyin, Cubic Kolmogorov Differential system with two limit cycles surrounding the same focus, Ann. of Diff. Eqs. 1(2) (1985), 201-207.

11. Wolfram Research Mathematica, A System for Doing Mathematics by Computer, Champaign, IL, (1988). 
Zhenhai Liu

Department of Mathematics,

Changsha University of Science and Technology

Changsha, Hunan 410077, China

E-mail: liuzh@mail.csuep.edu.cn

E. Sáez and I. Szántó

Departamento de Matemática, Universidad Ténica Federico Santa Mar'a

Casilla 110-V, Valparaíso - Chile.

E-mail: eduardo.saez@mat.utfsm.cl ivan.szanto@mat.utfsm.cl 